\title{
Comparison of the $\mathrm{HEI}$ and $\mathrm{HEI}-2010$ diet quality measures in association with chronic disease risk among low-income, African American urban youth in Baltimore, Maryland
}

\author{
Margaret M. Wrobleski, PhD, RDN and Elizabeth Parker, PhD, RD \\ University of Maryland School of Medicine, University of Maryland, Baltimore, Maryland.

\section{Kristen M. Hurley, PhD,} \\ Johns Hopkins Bloomberg School of Public Health, Johns Hopkins University, Baltimore, \\ Maryland.
}

\section{Sarah Oberlander, PhD, \\ U.S. Department of Health and Human Services, Washington, DC.}

Brian C. Merry, MPH, and

Yale University Center for Outcomes Research and Evaluation, Yale University, New Haven, CT.

\section{Maureen M. Black, PhD}

RTI International, Research Triangle Park, NC.

\section{Abstract}

Objective: Overall diet patterns may be a better predictor of disease risk than specific nutrients or individual foods. The purpose of this study is to examine how overall diet patterns relate to nutritional intake, body composition, and physiological measures of chronic disease risk among low-income, urban African American adolescents.

Methods: Cross-sectional data were collected from two samples of African American adolescents $(\mathrm{n}=317)$ from a low-income urban community, including dietary intake using the food frequency Youth/Adolescent Questionnaire and anthropometric measures. Serum cholesterol, serum lipoproteins, and glucose tolerance were measured in a subsample. Means testing compared differences in HEI and HEI-2010 component and total scores. Pearson correlations examined how HEI and HEI-2010 scores related to nutrient, food intakes, and markers of disease risk including body mass index, percent body fat, abdominal fat, serum cholesterol, serum lipoproteins and impaired glucose tolerance. Fisher R-Z transformations compared magnitude differences between HEI and HEI-2010 correlations to nutritional intake and chronic disease risk.

Results: Both HEI and HEI-2010 scores were positively associated with micronutrient intakes. Higher HEI scores were inversely related to serum cholesterol, low-density lipoprotein, impaired glucose tolerance, percent body fat and percent abdominal fat. HEI-2010 scores were not related to biomarkers of chronic disease risk.

Corresponding author: Margaret M. Wrobleski, PhD, RDN, University of Maryland School of Medicine, Growth and Nutrition Division, Phone: 252-588-0055, Peggy.Wrobleski@gmail.com. 
Conclusions: Compared to the HEI-2010, the HEI is a better indicator of chronic disease risk among low-income, urban African American adolescents.

\section{Keywords}

Adolescent; African American; Diet Quality; Minority Groups; Vulnerable Populations; HEI-2010

\section{INTRODUCTION}

A high quality diet during adolescence promotes healthy growth [1], yet the nutrient density of many adolescent diets is inadequate and adherence to nutritional guidelines is low [2,3]. Nutrient-poor dietary patterns formed during adolescence often track into adulthood [1] and increase the risk of obesity and chronic disease [4].

Few American adolescents consume a diet that meets the current recommendations in the 2015-2020 United States (US) Dietary Guidelines for Americans [5]. Nationwide surveys found that $8.5 \%$ of US high school students consumed the recommended servings of fruit, and only $2.1 \%$ ate adequate servings of vegetables [6]. Consuming fast food is linked to poorer diet quality and higher energy intake [7], yet one-third of all US children and adolescents are eating fast food on any given day [8]. National prevalence data indicate obesity among adolescents aged 12-19 years has been steadily trending upward from 1988 to 2014. Ogden et al., found significant racial differences in adolescent obesity with $22.6 \%$ of African American youth having a body mass index (BMI) percentile $295^{\text {th }}$ compared with $19.6 \%$ of white adolescents [9]. African American adolescents also ranked highest among extremely obese youth with $11.6 \%$ having a BMI $\geq 120 \%$ of the $95^{\text {th }}$ percentile, compared to $6.7 \%$ of white adolescents [9].

Adolescent racial weight disparities in the US appear to be related to poor diet [10]. Data from the 2011-2012 National Health and Nutrition Examination Survey (NHANES) indicate significant racial differences in the quality of adolescent diets, wherein African American adolescents had consistently lower diet quality scores (48.4 out of 100; $p=0.002)$ compared to white (50.2) or Hispanic (51.9) teenagers [11]. African American adolescents of low socioeconomic status (SES) are less likely to meet the recommended daily nutritional allowances, and eat significantly fewer servings of fruit, vegetables, dairy products and grains compared to higher SES white adolescents [12]. Low-income, African American adolescents' diets are disproportionately higher in energy-dense, nutrient-poor foods such as sweetened beverages, sweet/salty snacks, and fried foods [10, 12]. Excessive intake of energy-dense foods and dietary fat can lead to overweight, placing adolescents at risk for developing type 2 diabetes, hypertension, and cardiovascular disease (CVD) [13, 14]. Dietary intake associated with chronic disease risk among African American adolescents may partially explain why the racial health disparity gap widens as minority youth mature into adulthood [15].

Recent scientific advances in the multiple etiologies of chronic diseases and the health impact of synergistic interactions between nutrients and other food have led to increased interest in examining dietary patterns and disease [16, 17]. A growing area of research addresses relations between overall diet quality and health and disease, rather than the single 
nutrient-based approach to disease risk [17, 18]. Adherence to recommended healthful dietary patterns measured by diet quality indices, such as the Healthy Eating Index (HEI), has demonstarted the benefit of an overall healthful diet in disease prevention [19]. A number of diet quality indices have been developed based on the US Dietary Guidelines, although the efficacy of the indices predicting chronic disease risk or mortality has yet to be determined [18]. Of those studies investigating diet patterns and disease risk, very few have compared diet quality indices to biomarkers of chronic disease risk in minority youth populations.

The HEI measures diet quality by assessing how closely adults and children over the age of two years follow the 1995 US Dietary Guidelines for Americans [20]. Release of the 2010 US Dietary Guidelines for Americans [21] prompted the adoption of the Healthy Eating Index-2010 (HEI-2010) to estimate diet quality [22]. The HEI-2010 differs from the original HEI in that the scoring standards use a density approach, measuring dietary components as a percentage of total energy and nutritional intake, standardized per 1,000 kilocalories (kcals) [22]. In an attempt to measure diet quality over quantity, the HEI-2010 operationalizes diet quality by the relative proportion of recommended foods eaten rather than by the total quantity of foods consumed [22].

Among adult populations, HEI scores were weakly inversely associated with major chronic disease risk in men [23], but not in women [24]. Higher HEI scores were related to a reduced risk of all-cause mortality, cardiovascular, and cancer mortality in normal-weight adults with metabolic risk factors [25], and in British adults [26]. In a comparison of diet quality indices, Kant and Graubard found that the HEI was inversely related to biomarkers of chronic disease risk such as BMI, serum homocysteine (CVD-related risk indicator), plasma glucose and hemoglobin A1C (both biomarkers of type 2 diabetes risk) [27].

Higher HEI-2010 scores were associated with reduced risk of all-cause mortality and protective against cancer and CVD deaths in older adults [28], and in post-menopausal women compared to those in the lowest quintile of diet quality scores [29]. In an investigation combining data from three very large US cohort studies, Liese and colleagues found higher HEI-2010 scores were related to 11-28\% reductions in mortality due to all causes, CVD and cancer in adults [30]. Similarly, Li and colleagues found higher HEI-2010 scores were related to lower risk of liver cancer and fewer deaths resulting from chronic liver disease [31]. A large, multiethnic cohort comprised of diverse populations in the US compared various diet quality indices and found among African American adults, higher HEI-2010 scores were consistently associated with reductions in CVD deaths in men and women, and with decreased cancer deaths and all-cause mortality among African American men [32]. A similar inverse relation between HEI-2010 score and risk of disease deaths were reported among low-income African American adults in the Southeastern US, where the highest quintile of HEI-2010 scores were associated with a $20 \%$ lower risk of death from cancer, CVD, and other diseases [33].

There is scant research focusing on the HEI and HEI-2010 diet indices as indicators of chronic disease risk among adolescents. Physically active children consuming a healthy diet (operationalized as the top $40^{\text {th }}$ percentile of HEI score) had lower serum cholesterol, 
although among adolescents, there were no significant differences in biomarkers of disease risk and HEI scores [34]. There is a sizable gap in the literature describing the relationship between adolescents' diet quality and their risk of developing chronic disease, especially in nutritionally vulnerable, disadvantaged minority populations. A previous investigation by Hurley et al. comparing to the Youth Healthy Eating Index to the HEI, found the HEI was related to risk of chronic disease in low-income, urban African American adolescents [35]. This investigation will build upon the Hurley study and use the HEI as a comparison index to determine whether the HEI-2010 is associated with biomarkers of chronic disease risk.

The purpose of this study was to compare HEI and HEI-2010 total scores with nutritional intake, body composition, and physiological measures to determine the relative strength of each as indicators of chronic disease risk in low-income, African American urban youth. We hypothesized that compared to HEI scores: 1) higher HEI-2010 total scores are associated with higher micronutrient intake and fewer servings of snack/desserts and sweetened beverages which would be protective factors against developing chronic disease; and 2) HEI-2010 scores are inversely correlated to biomarkers of chronic disease risk, such as BMI, elevated percent body fat and abdominal fat, serum cholesterol, LDL, and impaired glucose tolerance.

\section{MATERIAL AND METHODS}

\section{Participants}

Analyses were based on data from two samples of low-income, urban African American adolescent participants from two longitudinal, randomized controlled intervention trials designed to promote either healthy diet and physical activity behaviors among middle school students [36] (Challenge $\mathrm{N}=235$ ) or positive parenting practices and delay of second births among adolescent mothers [37] (Three Generation Project, N=121). The University of Maryland Institutional Review Board approved study protocols. Signed assent or informed consent forms, demographic, and income data were obtained from all participants (and parents of participants under 18 years) and participants were compensated for evaluation visits. Low-income was defined as family income $<185 \%$ of poverty level.

For the Challenge study, adolescent recruitment sites included three urban public middle schools and a primary care facility [36]. Eligibility criteria included age 11-16 years. There was no mention of weight status or obesity in recruitment procedures. Baseline measures were collected from April 2001 through May 2004, prior to randomization into intervention groups. Seven participants identifying themselves as non-African American and 32 participants reporting implausible dietary intake $(<500$ or $>5,000 \mathrm{kcal})$ [38] were excluded from the original sample $(\mathrm{n}=196,83 \%)$ for this analysis.

For the Three Generation Project, adolescent mothers were approached shortly after delivery and recruited into the study from three urban hospitals between June 1997 and September 1999 [37]. Eligibility criteria included maternal age less than 18 years, low-income (defined as eligible for the Special Supplemental Nutrition Program for Women, Infants, and Children (WIC), or having a family income $<185 \%$ of poverty level), African American, primiparous, and no identified mental illness or chronic condition that would interfere with 
parenting or normal adolescent development. Data reported here were collected at 13 months postpartum, representing two thirds $(\mathrm{n}=121,67 \%)$ of the original sample [37]. In this sample, nine participants were either lactating $(n=3)$ or pregnant $(n=6)$ but since there were no differences in BMI, HEI, or HEI-2010 scores by pregnancy/lactation status, these participants were included in the analysis. Adolescent mothers reported lactation status at baseline data collection, but not during the time period assessed by the food frequency questionnaire (FFQ). We found no statistical difference in HEI or HEI-2010 scores by intervention status; therefore, intervention groups were combined.

\section{Measures}

Dietary intake was measured using the Youth/Adolescent Questionnaire (YAQ), a selfadministered, validated FFQ specifically developed for older children and adolescents [39]. Adolescents reported dietary intake over the past 12 months using the 131-item YAQ. Nutritional analysis produced estimates for energy, macronutrients, micronutrients, and mean daily servings of foods sorted into seven broad categories: beverages, dairy products, main dishes, miscellaneous foods like gravy and condiments, breads and cereals, fruits and vegetables, snack foods/desserts. Sweetened beverages such as soda and snack foods/ desserts were used in the analysis to represent energy-dense, nutrient-poor foods that can contribute to obesity and increase chronic disease risk.

HEI scores reflect adherence to serving guidelines represented in the 1992 Food Guide Pyramid and recommendations for limiting dietary cholesterol, sodium intake, total fat and saturated fat consumption, and including a variety of foods in the diet [20]. The HEI estimates diet quality using ten component scores, each ranging from zero to ten. Five components measure nutritional intake of fruits, vegetables, grains, milk, and meat. The remaining components assess dietary cholesterol, sodium, total fat, saturated fat as a percentage of total caloric intake, and dietary variety [20]. Since dietary recommendations differ across gender and age groups, separate scoring algorithms are required for age and gender when calculating the component scores for food consumption [20]. HEI scores range from $0-100$, with higher HEI scores reflecting better diet quality. A detailed description of the HEI has been published elsewhere [35].

The HEI-2010 consists of twelve components that are summed for the total HEI-2010 score (Table 1). The components reflect the 2010 US Dietary Guidelines for Americans that include nine adequacy components (total fruit, whole fruit, total vegetables, greens and beans [dark green vegetables and legumes], whole grains, dairy, total protein foods, seafood and plant proteins, and fatty acids), and three moderation components (refined grains, sodium, and empty calories from solid fat, alcohol, and added sugars) [22]. Each component score within the HEI-2010 calculates the degree of adherence to specific dietary recommendations, with scores ranging from zero for lack of compliance up to the maximum component's points for full dietary compliance (Table 1). The HEI-2010 total score can range from zero as the lowest score possible to a high score of 100, indicating an ideally nutritious diet [22]. HEI-2010 scores are based on the cup or ounce equivalent per 1,000 kcal for most scores [22] (Table 1). Higher HEI-2010 scores reflect better dietary compliance with the 2010 Dietary Guidelines for Americans. 
Trained staff collected anthropometric data from adolescents using identical measurement protocols across the two studies. Height was measured using a wall-mounted stadiometer to the nearest $0.5 \mathrm{~cm}$ and weight was measured with a (Tanita) digital scale to the nearest 100 g. BMI values calculated as $\mathrm{kg} / \mathrm{m}^{2}$ were converted to percentiles and Z-scores based on the 2000 Centers for Disease Control and Prevention's age- and gender-specific tables and algorithms [40]. Adolescents were categorized as normal weight ( $<85^{\text {th }}$ percentile), overweight $\left(\geq 85^{\text {th }}\right.$ percentile and $<95^{\text {th }}$ percentile), or obese $\left(\geq 95^{\text {th }}\right.$ percentile).

A subset of Challenge participants was measured for dual energy x-ray absorptiometry (DXA) body composition, serum cholesterol, LDL, and glucose tolerance. The General Clinical Research Center (GCRC) at the University of Maryland in Baltimore was not established until late in the Challenge recruitment process, so these baseline measures could only be collected from study participants recruited after the GCRC opened. Body composition was measured using DXA by trained technicians. Research technicians measured body composition following standardized protocols using a Hologic QDR $4500 \mathrm{~W}$ scanner and used the manufacturer's software to calculate abdominal fat and body fat. Abdominal fat was defined as the percentage of fat contained within the area measured laterally between the second lumbar vertebra and the fourth lumbar vertebra, and bounded vertically by the lateral inferior borders of the rib cage and the iliac crest.

Physiological measures included collection of blood samples and a fasting two-hour oral glucose tolerance test from a subsample of Challenge participants immediately following the DXA scan [41]. Serum cholesterol $(\mathrm{n}=58)$, HDL and LDL $(\mathrm{n}=55)$, and glucose tolerance $(n=57)$ were measured. The glucose tolerance procedure followed the American Diabetes Association regiment using pediatric dosing guidelines. Impaired glucose tolerance was defined as a fasting serum glucose level from $140 \mathrm{mg} / \mathrm{dL}$ to $199 \mathrm{mg} / \mathrm{dL}$ at two hours post ingestion of glucose load [42]. A detailed description of the glucose tolerance test and body composition testing on this sample has been published elsewhere [41].

\section{Analysis}

The two samples used in this investigation were merged to create a combined study sample to compare total HEI/HEI-2010 score by nutrient intake, sweetened beverage and snack consumption. Participants in both samples were from the same Baltimore city neighborhoods and shared a similar socioeconomic background. Dietary YAQ data were used to calculate the HEI and HEI-2010 component and total scores. Details related to how the HEI was coded using the YAQ are documented elsewhere [35]. For the HEI-2010, we modified the US Department of Agriculture (USDA) scoring algorithm to assess diet quality using food frequency data from the YAQ rather than from 24-hour dietary recall data typically used to compute HEI-2010 component and summary diet quality scores. To accomplish this, we matched each food item at the specified serving size on the YAQ to a food identifier code in the USDA Food and Nutrient Database [43]. Then each survey food's weight was calculated in grams and converted into MyPyramid-equivalents. We adapted publically available SAS programming codes (see https://epi.grants.cancer.gov/hei/ tools.html\#sas) from the National Cancer Institute to transform the food frequency items into HEI-2010 component and summary scores [22]. 
We used $t$-tests to analyze differences in total and component mean HEI and HEI-2010 scores by sample. Using Pearson correlations, we examined the relations between the HEI and HEI-2010 total scores with micronutrient (iron, folate, calcium and vitamin A) intake, energy, fiber, sweetened beverages, and snacks/desserts intake. We also correlated HEI and HEI-2010 total scores with BMI z-score percentiles, percent body fat, percent abdominal fat, serum cholesterol, HDL, LDL, and impaired glucose tolerance to determine whether the HEI-2010 was more closely associated with indicators of chronic disease risk than the HEI. Fisher R-Z transformations indicated magnitude differences between HEI and HEI-2010 correlations to nutritional intake and physiological biomarkers of chronic disease risk.

We analyzed data using SAS (SAS 9.4 RTI International, Research Triangle Park, NC, 2015) and SPSS statistical software (SPSS version 22.0.0, SPSS, Inc., Chicago, IL, 2013), set a significance level of 0.05 , corrected for multiple comparisons, and reported values as means with standard deviations. Pearson correlations and Fisher R-Z transformation $p$-values for dietary intake variables were adjusted using Bonferroni multiple comparison post hoc testing. With eight pairs of correlations, the cutoff for significance was set at $p<0.006$ instead of $p<0.05$.

\section{RESULTS}

The mean age of the Challenge sample was 13.2 years, with gender evenly split (49\% female). Fifty-six percent of participants' families were living at or below the poverty line; $12 \%$ of participants were overweight and $27 \%$ were obese. The mean age of the Three Generation sample was 17.5 years, $22 \%$ were overweight and 35\% were obese (Table 2). BMI z-score distributions in both the Challenge and Three Generation samples exceeded national estimates [9], with the Three Generation Project adolescents having significantly higher BMI z-scores $(1.0 \pm 1.08$ vs. $0.7 \pm 1.15 ; p=0.03)$.

\section{HEI/HEI-2010 Scores by Gender}

The majority (69\%) of adolescents across the combined sample were female $(n=220)$. Male adolescents had higher HEI-2010 total scores compared to females (55.1 \pm 8.30 vs. 53.0 $\pm 8.59 ; p=0.04$ ), although HEI total score did not vary by gender. Male adolescents had higher HEI-2010 component scores than females for dairy $(8.3 \pm 2.16$ vs. $6.8 \pm 2.64 ; p<$ $0.001)$ and fatty acid ratios ( $3.6 \pm 2.07$ vs. $2.0 \pm 2.41 ; p<0.001)$, and adolescent females had higher component scores for total vegetables ( $2.9 \pm 1.01$ vs. $2.6 \pm 1.02 ; p<0.001)$ and empty calories ( $8.9 \pm 4.11$ vs. $7.7 \pm 4.20 ; p=0.02)$ compared to their male counterparts. Finally, folate intake was higher in male adolescents compared to females $(431 \pm 215.1$ vs. 367 $\pm 207.1 ; p=0.02$ ).

\section{HEI/HEI-2010 Scores by Sample}

Micronutrient intake of iron and vitamin A among participants in both samples met Adequate Intake and Recommended Dietary Allowance values [44] for adolescents. Dietary intake of fiber and calcium was found to be inadequate across both samples, and folate intake among Three Generation participants was deficient [44]. Total HEI-2010 scores in the Challenge sample exceeded those from the Three Generation sample (55.8 \pm 8.23 vs. 50.3 
$\pm 7.95 ; p<0.001)$, as did HEI total scores (62.8 \pm 11.75 vs. $59.9 \pm 11.27 ; p<0.05)$ (Table 2). The Challenge sample had higher HEI-2010 component scores for whole grains, dairy, and fatty acid ratios compared to the Three Generation sample (Table 1). The HEI component score for dairy was higher in the Challenge sample $(7.1 \pm 2.79$ vs. $6.2 \pm 3.01 ; p<0.01)$ than in the Three Generation sample. In the combined sample, overall HEI score was higher than total HEI-2010 score $(61.7 \pm 11.64$ vs. $53.7 \pm 8.54 ; p<0.001)$.

\section{$\mathrm{HEl} / \mathrm{HEI}-2010$ Scores and Health Indicators}

There were significant positive correlations using Bonferroni adjusted $p$-values for both the HEI and the HEI-2010 between total index scores and dietary iron, fiber, folate, and vitamin A (Table 3). The HEI total score was weakly correlated with sweetened beverages ( $\mathrm{r}=0.18$; $p<0.001)$ and moderately related to snacks/desserts $(\mathrm{r}=0.40 ; p<0.001)$ and energy intake $(\mathrm{r}=0.58 ; p<0.001)$. The HEI-2010 total score had a moderate, inverse relationship to sweetened beverages $(\mathrm{r}=-0.31 ; p<0.001)$ and a weak, inverse association with snacks/ desserts $(\mathrm{r}=-0.12 ; p<0.03)$. The magnitude of the correlation with the HEI was significantly stronger than with the HEI-2010 for vitamin A intake (Table 3).

HEI scores were inversely correlated to percent body fat, abdominal fat, serum cholesterol, LDL, and impaired glucose tolerance, although HEI-2010 scores were not. Overweight and obese BMI percentiles were not associated with either HEI or HEI-2010 (Table 3).

\section{DISCUSSION}

This investigation into the comparison of two measures of diet quality in two samples of African American adolescents yielded four main findings. First, across both samples, the HEI and HEI-2010 scores reflect suboptimal diet quality in nutritional intake. Adolescents across both samples had inadequate dietary intakes of calcium and fiber, and the Three Generation young mothers had insufficient folate intake [44]. Excepting calcium, intakes of select micronutrients important to healthy adolescent growth were correlated with the HEI and HEI-2010, suggesting that both measures are sensitive to nutritional adequacy and deficiencies. All Baltimore public schools provided free school lunches at the time of baseline data collection. Since all Challenge participants and some Three Generation adolescent mothers attended school during the study, the consumption of school lunches may have positively contributed to overall adolescent diet quality scores. Diet quality scores may also have been higher among Three Generation adolescent mothers who were participating in WIC, although WIC enrollment information was not collected at baseline.

Second, HEI scores exceeded HEI-2010 scores. One possible explanation is that the HEI does not standardize energy intake and can inflate diet quality estimates in individuals with high dietary variety due to excessive energy consumption. Unlike the HEI-2010 [22], the HEI score credits consumption of all foods and does not discount points for less nutritious foods in the diet [20]. This aspect of the HEI scoring algorithm may explain why higher HEI scores were positively related to consumption of energy, snacks/desserts, and sweetened beverages, while the HEI-2010 had an inverse relation to snacks/desserts and sweetened beverage consumption. We did not see nor expect to find energy consumption correlated to HEI-2010 scores since this index measure is standardized for caloric intake [22]. 
Third, the difference in scoring rubrics may have influenced findings. Total score values of the older HEI were designed to classify dietary intake patterns as "poor," "needs improvement," or "good" quality [20]. Unlike the original HEI, HEI-2010 total score does not rate diet quality as such. Alternatively, a comparison of component scores between these two indices would not be feasible because the $10 \mathrm{HEI}$ dietary components are not identical to the 12 nutritional components used in the HEI-2010 [20, 22]. Due to the inherent differences in scoring rubrics, assessing risk of chronic disease by correlating HEI-2010 total score with physiological disease risk factors may not be an optimal analytical approach for this index.

Finally, the HEI's inverse association to adiposity and physiological risk factors suggest that the HEI was better than the HEI-2010 in identifying individuals at risk for chronic disease, particularly for cardiometabolic risk factors associated with cardiovascular disease and type 2 diabetes. This finding confirms the HEI's association to metabolic biomarkers of chronic disease risk found among adults in previous research [23, 25-27]. One possible explanation why the HEI-2010 was not related to biomarkers of disease risk is because this index controls for energy consumption [22], and the associations between excessive energy intake and indicators of disease risk such as adiposity and pathophysiologic metabolic markers, may be suppressed in the HEI-2010. The reliance on a nutrient density approach that discounts energy intake [22] may, in part, explain why the HEI-2010 was not related to biomarkers of chronic disease risk. The strength and significance of this paper is that it is essentially comparing the efficacy of estimating chronic disease risk by comparing diet quality indices that either use a standardized caloric density approach or measure the actual quantity of food consumed. The HEI-2010 measures diet quality over quantity, and operationalizes diet quality by the relative proportion of recommended foods eaten; the HEI is a more direct assessment of diet quality based on the total quantity of foods consumed. It is possible that the underlying structure of the diet quality index in regards to measuring caloric quality versus food quantity is the key to the index's ability to predict chronic disease risk in adolescents.

The current 2015-2020 US Dietary Guidelines differs from earlier versions by adding recommendations for making behavioral shifts in improving eating habits and suggestions for improving dietary intake in a variety of environmental and social settings [45]. Although the dietary guidelines are updated, a corresponding diet quality index to replace the HEI-2010 has not yet been released. Research has shown the HEI-2010 is a useful instrument for associating reduced chronic disease mortality risk with diet quality in adults [28-31] and in African American populations [32,33]. These studies agree that higher HEI-2010 scores are associated with reduced chronic disease mortality in adults and signify greater adherence to the 2010 Dietary Guidelines for Americans [21].

The HEI-2010 was used in this study because it is the most current HEI version available to assess diet quality. The Alternative Healthy Eating Index (AHEI) was not deemed appropriate for use in this study since it awards diet quality points for moderate alcohol intake and consumption of cereal fiber [46], both of which were not measured by the FFQ used in this research. Since the alcohol component of the AHEI makes a substantial statistical contribution to overall diet quality score and has been shown to be a major 
predictor of cardiovascular and all-cause mortality [46], measurement bias might have influenced results if the AHEI was used in this investigation to estimate diet quality in minority youth.

Determining which diet quality index to use when estimating chronic disease risk will vary, depending on whichever instrument has been proven most appropriate for a given sample population [47]. Compared to the HEI in this investigation, the HEI-2010 appears to have lower sensitivity to detect associations related to chronic disease risk in disadvantaged, urban African American youth, even though it has been associated with chronic disease risk in adult African American population samples [32, 33]. When using diet pattern data from low-income, African American urban adolescents, this investigation found that the HEI was better at estimating chronic disease risk compared to the HEI-2010.

A few study limitations should be considered. First, the cross sectional design limits causal interpretations. Second, the use of self-reported dietary data recounted by adolescents on the YAQ may possibly have introduced misclassification. Finally, relations between diet quality measures and physiological indicators of disease were limited to a relatively small subsample of adolescents, thereby restricting power to detect a significant statistical correlation between the HEI-2010 and biomarkers of disease risk.

\section{CONCLUSION}

The diet quality of minority adolescents falls short of nutritional recommendations [2] and is of concern because of the increased risk of chronic disease in adulthood [4]. Accurately measuring diet quality remains a critical element in nutrition monitoring efforts. Adolescent diet quality is a multifaceted, complex concept that involves a cultural understanding of influences that shape dietary behaviors. Research has shown that African American adolescents have suboptimal nutritional intake [11], especially in low-income urban communities where there is limited availability of affordable, healthful foods [48]. Poor diet quality coupled with a 30-year increasing trend in extreme obesity prevalence among African American adolescents [9] has resulted in higher rates of chronic disease in African Americans compared to other ethnic population samples [49].

Evidence of cardiovascular risk factors and early development of chronic disease related to adiposity in pre-adolescence children continuing into young adulthood [50] is concerning. Nutrient-poor adolescent dietary patterns among females have predicted clustering of cardiovascular risk factors by the end of adolescence, although girls with healthier diet patterns had significantly less cardiometabolic risk accumulation [14], indicating improved diet quality reduces chronic disease risk. The disproportionately poor diet quality of lowincome African American adolescents [10,15] is a pressing public health concern evidenced by cardiometabolic risk increases in youth [50] and by elevated risk for metabolic dyslipidemia in non-Hispanic African American adolescents [51]. Future research efforts are needed to identify a diet quality index for disadvantaged African American adolescent populations that can be validated to predict chronic disease risk. To date, the HEI is a better indicator of chronic disease risk among low-income, urban African American adolescents compared to the HEI-2010. 


\section{ACKNOWLEDGMENTS}

The research culminating in this manuscript was supported by grants R40MC00241, MCJ-240301, and R40MC04297 from the Maternal and Child Health Research Program, US Department of Health and Human Services; grant APRPA006000 from the Office of Population Affairs, US Department of Health and Human Services, the University of Maryland General Clinical Research Center; and grant M01 RR16500 General Clinical Research Centers Program, National Center for Research Resources (NCRR), NIH.

\section{REFERENCES}

1. Story M, Neumark-Sztainer D, French SA: Individual and environmental influences on adolescent eating behaviors. J Am Diet Assoc 102(3 Suppl):S40-51, 2002.

2. Hiza HAB, Guenther PM, Rihane CI: Diet quality of children age 2-17 years as measured by the Healthy Eating Index-2010. Washington (DC): United States Department of Agriculture; 2013 http://www.cnpp.usda.gov/Publications/NutritionInsights/Insight52.pdf. Accessed June 20, 2016.

3. Banfield EC, Liu Y, Davis JS, Chang S, Frazier-Wood AC: Poor adherence to US Dietary Guidelines for children and adolescents in the NHANES population. J Acad Nutr Diet 116(1):21-27, 2016. [PubMed: 26391469]

4. Short KR, Blackett PR, Gardner AW, Copeland KC: Vascular health in children and adolescents: effects of obesity and diabetes. Vasc Health Risk Manag 5:973-990, 2009. [PubMed: 19997578]

5. United States Department of Agriculture and United States Department of Health and Human Services. 2015-2020 Dietary Guidelines for Americans. 8th Edition. December 2015. Washington (DC): US Government Printing Office; 2015 Available at https://health.gov/dietaryguidelines/2015/ guidelines. Accessed May 20, 2017.

6. Moore LV, Thompson FE, Demissie Z: Percentage of youth meeting federal fruit and vegetable intake recommendations, Youth Risk Behavior Surveillance System, United States and 33 states, 2013. J Acad Nutr Diet 117:545-553, 2017. [PubMed: 27988220]

7. Powell LM, Nguyen BT: Fast-food and full-service restaurant consumption among children and adolescents: effect on energy, beverage, and nutrient intake. JAMA Pediatr 167(1):14-20, 2013. [PubMed: 23128151]

8. Vikraman S, Fryar CD, Ogden CL: Caloric intake from fast food among children and adolescents in the United States, 2011-2012 NCHS Data Brief. No 213. Hyattsville (MD): National Center for Health Statistics, 2015.

9. Ogden CL, Carroll MD, Lawman HG, Fryar CD, Kruszon-Moran D, Kit BK, Flegal KM: Trends in obesity prevalence among children and adolescents in the United States, 1988-1994 through 20132014. JAMA 315(21):2292-2299, 2016. [PubMed: 27272581]

10. Wang Y, Jahns L, Tussing-Humphreys L, Xie B, Rockett H, Liang H, Johnson L: Dietary intake patterns of low-income urban African American adolescents. J Am Diet Assoc 110:1340-1345, 2010. [PubMed: 20800126]

11. Gu X, Tucker KL: Diet quality of the US child and adolescent population: Trends from 1999 to 2012 and associations with the use of federal nutrition assistance programs. Am J Clin Nutr 105:194-202, 2017. [PubMed: 27881390]

12. Fahlman MM, McCaughtry N, Martin J, Shen B: Racial and socioeconomic disparities in nutrition behaviors: Targeted interventions needed. J Nutr Educ Behav 42:10-16, 2010. [PubMed: 19910257]

13. Juonala M, Magnussen CG, Berenson GS, Venn A, Burns TL, Sabin MA, Srinivasan SR, Daniels SR, Davis PH, Chen W, Sun C, Cheung M, Viikari JS, Dwyer T, Raitakari OT: Childhood adiposity, adult adiposity, and cardiovascular risk factors. N Engl J Med 365:1876-1885, 2011. [PubMed: 22087679]

14. Moore LL, Singer MR, Bradlee ML, Daniels SR: Adolescent dietary intakes predict cardiometabolic risk clustering. Eur J Nutr, 2 28, 2016 DOI 10.1007/s00394-015-0863-8. Accessed November 29, 2015.

15. Kirkpatrick SI, Dodd KW, Reedy J, Krebs-Smith SM: Income and race/ethnicity are associated with adhereance to food-based dietary guidance among US adults and children. J Acad Nutr Diet 112(5):624-635, 2012. [PubMed: 22709767] 
16. Hu FB: Dietary pattern analysis: A new direction in nutritional epidemiology. Curr Opin Lipidol 13(1):3-9, 2002. [PubMed: 11790957]

17. Tucker K: Dietary patterns, approaches, and multicultural perspective. Appl Physiol Nutr Metab 35:211-218, 2010. [PubMed: 20383235]

18. Alkerwi A, Vernier C, Crichton GE, Sauvageot N, Shivappa N, Hebert JR: Cross-comparison of diet quality indices for predicting chronic disease risk: findings from the Observation of Cardiovascular Risk Factors in Luxembourg (ORISCAV-LUX) study. Br J Nutr 113(2):259-269, 2015. [PubMed: 25475010]

19. Schwingshackl L, Hoffmann G: Diet quality as assessed by the Healthy Eating Index, the Alternate Healthy Eating Index, the Dietary Approaches to Stop Hypertension Score, and health outcomes: A systematic review and meta-analysis of cohort studies. J Acad Nutr Diet 115:780-800, 2015. [PubMed: 25680825]

20. Kennedy ET, Ohls J, Carlson S, Fleming K: The Healthy Eating Index: Design and applications. J Am Diet Assoc 5(10):1103-1108, 1995.

21. U.S. Department of Agriculture and U.S. Department of Health and Human Services. Dietary Guidelines for Americans, 2010. 7th Edition. Washington, DC: US Government Printing Office, December 2010. Available at https://www.cnpp.usda.gov/sites/default/files/ dietary_guidelines_for_americans/PolicyDoc.pdf. Accessed August 22, 2017.

22. Guenther PM, Casavale KO, Reedy J, Kirkpatrick SI, Hiza HA, Kuczynski KJ, Kahle LL, KrebsSmith SM: Update of the Healthy Eating Index: HEI-2010. JAcad Nutr Diet 113(4):569-580, 2013. [PubMed: 23415502]

23. McCullough ML, Feskanich D, Rimm EB, Giovannucci EL, Ascherio A, Variyam JN, Spiegelman D, Stampfer MJ, Willett WC: Adherence to the Dietary Guidelines for Americans and the risk of major chronic disease in men. Am J Clin Nutr 72:1223-1231, 2000. [PubMed: 11063453]

24. McCullough ML, Feskanich D, Stampfer MJ, Rosner BA, Hu FB, Hunter DJ, Variyam JN, Colditz GA, Willett WC: Adherence to the Dietary Guidelines for Americans and the risk of major chronic disease in women. Am J Clin Nutr 72:1214-1222, 2000. [PubMed: 11063452]

25. Park YM, Fung TT, Steck SE, Zhang J, Hazlett LJ, Han K, Lee SH, Merchant AT: Diet quality and mortality risk in metabolically obese normal-weight adults. Mayo Clin Proc 91(10):1372-1383, 2016. [PubMed: 27712636]

26. Ovani S, Haghighatdoost F, Surkan PJ, Larijani B, Azadbakht L: Adherence to the Healthy Eating Index and Alternative Healthy Eating Index dietary patterns and mortality from all causes, cardiovascular disease and cancer: a meta-analysis of observational studies. J Hum Nutr Diet 30(2):216-226, 2017. [PubMed: 27620213]

27. Kant AK, Graubard BI: A comparison of three dietary pattern indexes for predicting biomarkers of diet and disease. J Am Coll Nutr 24(4):294-303, 2005. [PubMed: 16093407]

28. Reedy J, Kreb-Smith SM, Miller PE, Liese AD, Kahle LL, Park Y, Subar AF: Higher diet quality is associated with decreased risk of all-cause, cardiovascular disease, and cancer mortality among older adults. J Nutr 144:881-889, 2014. [PubMed: 24572039]

29. George SM, Ballard-Barbash R, Manson JE, Reedy J, Shikany JM, Subar AF, Tinker LF, Vitolins $\mathrm{M}$, Neuhouser ML: Comparing indices of diet quality with chronic disease mortality risk in postmenopausal women in the Women's Health Initiative Observational Study: Evidence to inform national dietary guidance. Am J Epidemiol 180(6):616-625, 2014. [PubMed: 25035143]

30. Liese AD, Krebs-Smith SM, Subar AF, George SM, Harmon BE, Neuhouser ML, Boushey CJ, Schap TE, Reedy J: The Dietary Patterns Methods Project: Synthesis of findings across cohorts and relevance to dietary guidance. J Nutr 145:393-402, 2015. [PubMed: 25733454]

31. Li WQ, Park Y, McGlynn KA, Hollenbeck AR, Taylor PR, Goldstein AM, Freedman ND: Indexbased dietary patterns and risk of incident hepatocellular carcinoma and mortality from chronic liver disease in a prospective study. Hepatology 60:588-597, 2014. [PubMed: 24715615]

32. Harmon BE, Boushey CJ, Shvetsov YB, Ettienne R, Reedy J, Wilkens LR, Marchand LL, Henderson BE, Kolonel LN: Associations of key diet-quality indexes with mortality in the Multiethnic Cohort: The Dietary Patterns Methods Project. Am J Clin Nutr 101:587-597, 2015. [PubMed: 25733644] 
33. Yu D, Sonderman J, Buchowski MS, McLaughlin JK, Shu XO, Steinwandel M, Signorello LB, Zhang X, Hargreaves MK, Blot WJ, Zheng W: Healthy eating and risks of total and cause-specific death among low-income populations of African-Americans and other adults in the Southeastern United States: A prospective cohort study. PloS Med 12(5):e1001830, 2015. [PubMed: 26011727]

34. Loprinzi PD, Lee IM, Andersen RE, Crespo CJ, Smit E: Association of concurrent healthy eating and regular physical activity with cardiovascular disease risk factors in US youth. Am J Health Promot 30(1):2-8, 2015. [PubMed: 25372232]

35. Hurley KM, Oberlander SE, Merry BC, Wrobleski MM, Klassen AC, Black MM: The HEI and YHEI are unique, non-redundant measures of diet quality among low-income, African American adolescents. J Nutr 139(2):359-364, 2009. [PubMed: 19074210]

36. Black MM, Hager ER, Le K, Anliker J, Arteaga SS, Diclemente C, Gittelsohn J, Madger L, Papas M, Snitker S, Treuth MS, Wang Y: Challenge! Health promotion/obesity prevention mentorship model among urban, black adolescents. Pediatrics 126(2):280-288, 2010. [PubMed: 20660556]

37. Black MM, Bentley ME, Papas MA, Oberlander S, Teti LO, McNary S, Le K, O’Connell M: Delaying second births among adolescent mothers: a randomized, control trial of a home-based mentoring program. Pediatrics 118(4):1087-1099, 2006. [PubMed: 16951002]

38. Willett WC: "Nutritional Epidemiology," 2nd edition. New York: Oxford University Press; p. 322, 1998.

39. Rockett HRH, Breitenbach M, Frazier AL, Witschi J, Wolf AM, Field AE, Colditz GA: Validation of a youth/adolescent food frequency questionnaire. Prev Med 26:808-816, 1997. [PubMed: 9388792]

40. 2000 Growth Charts for the United States: methods and development. Atlanta (GA): Centers for Disease Control; 2002 http://www.cdc.gov/nchs/data/series/sr_11/sr11_246.pdf. Accessed July 28, 2011.

41. Snitker S, Le KY, Hager ER, Caballero B, Black MM: Association of physical activity and body composition with insulin sensitivity in a community sample of adolescents. Arch Pediatr Adolesc Med 161(7):677-683, 2007. [PubMed: 17606831]

42. Diagnosis and classification of diabetes mellitus. Alexandria (VA): American Diabetes Association; Diabetes Care 32 Suppl 1:S62-S67, 2009.

43. Food and Nutrient Database for Dietary Studies, 1.0. Beltsville (MD): United States Department of Agriculture, Agriculture Research Service, Food Surveys Research Group; 2004.

44. Dietary Reference Intakes: The essential guide to nutrient requirements. Washington (DC): National Academy of Sciences, Institute of Medicine; 2006 http://www.nap.edu/read/11537/ chapter/1\#ii. Accessed June 20, 2016.

45. United States Department of Health and Human Services. Health.gov: Top 10 things you need to know about the 2015-2020 Dietary Guidelines for Americans. Washington, (DC): Office of Disease Prevention and Health Promotion; 2017 https://health.gov/news/dietary-guidelines-digitalpress-kit/2016/01/top-10-things-you-need-to-know.htm. Accessed on February 3, 2017.

46. Akbaraly TN, Ferrie JE, Berr C, Brunner EJ, Head J, Marmot MG, Singh-Manoux A, Ritchie K, Shipley MJ, Kivimaki M: Alternative Healthy Eating Index and mortality over 18 y of follow-up: Results from the Whitehall II cohort. Am J Clin Nutr 94:247-53, 2011. [PubMed: 21613557]

47. Guenther PM, Kirkpatrick SI, Reedy J, Krebs-Smith SM, Buckman DW, Dodd KW, Casavale KO, Carroll RJ: The Healthy Eating Index-2010 is a valid and reliable measure of diet quality according to the 2010 Dietary Guidelines for Americans. J Nutr 144:399-407, 2014. [PubMed: 24453128]

48. Bower KM, Thorpe RJ, Rohde C, Gaskin DJ: The intersection of neighborhood racial segregation, poverty, and urbanicity and its impact on food store availability in the United States. Prev Med 58:33-39, 2014. [PubMed: 24161713]

49. Daw J: Contribution of four comorbid conditions to racial/ethnic disparities in mortality risk. Am J Prev Med 52(1S1):S95-S102, 2017. [PubMed: 27989298]

50. Chen W, Bao W, Begum S, Elkasabany A, Sirinivasan SR, Berenson GS: Age-related patterns of the clustering of cardiovascular risk variables of syndrome $\mathrm{X}$ from childhood to young adulthood in a population made up of black and white subjects: the Bogalusa Heart Study. Diabetes 49(6): 1042-1048, 2000. [PubMed: 10866058] 
51. Gidding SS, Keith SW, Falkner B: Adolescent and adult African Americans have similar metabolic dyslipidemia. J Clin Lipidol 9(3):368-376, 2015. [PubMed: 26073396] 
Table 1.

HEI-2010 Scoring criteria and HEI-2010 mean component and total scores for Challenge, Three Generation adolescents, and Combined Sample $(\mathrm{N}=317)$

\begin{tabular}{|c|c|c|c|c|c|c|}
\hline \multicolumn{4}{|l|}{ HEI-2010 Scoring Criteria } & \multicolumn{3}{|c|}{ HEI-2010 Scores, mean (range) } \\
\hline HEI-2010 Component & Maximum points & $\begin{array}{l}\text { Standard for } \\
\text { minimum score of } \\
\text { 0 }\end{array}$ & $\begin{array}{l}\text { Standard for } \\
\text { maximum } \\
\text { score }\end{array}$ & $\begin{array}{l}\text { Challenge }(n \\
=196)\end{array}$ & $\begin{array}{l}\text { Three } \\
\text { Generation } \\
(\mathbf{n}=\mathbf{1 2 1})\end{array}$ & $\begin{array}{l}\text { Combined } \\
\text { Sample (N = } \\
\text { 317) }\end{array}$ \\
\hline Total Fruit & 5 & No Fruit & $\begin{array}{l}\geq 0.8 \text { cup } \\
\text { equiv. per } \\
1,000 \text { kcals }\end{array}$ & $3.6(0-5)$ & $3.6(0-5)$ & $3.6(0-5)$ \\
\hline Whole Fruit & 5 & No Whole Fruit & $\begin{array}{l}\geq 0.4 \text { cup } \\
\text { equiv. per } \\
1,000 \text { kcals }\end{array}$ & $3.7(0-5)$ & $3.4(0-5)$ & $3.6(0-5)$ \\
\hline Total Vegetables & 5 & No Vegetables & $\begin{array}{l}\geq 1.1 \text { cup } \\
\text { equiv. per } \\
1,000 \text { kcals }\end{array}$ & $2.8(0-5)$ & $2.9(1-5)$ & $2.8(0-5)$ \\
\hline Greens and Beans & 5 & $\begin{array}{l}\text { No Dark-green } \\
\text { vegetables or } \\
\text { Legumes }\end{array}$ & $\begin{array}{l}\geq 0.2 \text { cup } \\
\text { equiv. per } \\
1,000 \text { kcals }\end{array}$ & $1.8(0-5)$ & $1.8(0-5)$ & $1.8(0-5)$ \\
\hline Whole Grain * & 10 & No Whole Grains & $\begin{array}{l}\geq 1.5 \text { oz equiv. } \\
\text { per } 1,000 \text { kcals }\end{array}$ & $2.1(0-10)$ & $1.7(0-8)$ & $1.9(0-10)$ \\
\hline Dairy $* *$ & 10 & No Dairy & $\begin{array}{l}\geq 1.3 \text { cup equiv. } \\
\text { per } 1,000 \text { kcals }\end{array}$ & $7.9(2-10)$ & $6.1(1-10)$ & $7.2(2-10)$ \\
\hline Total Protein Foods & 5 & No Protein Foods & $\begin{array}{l}\geq 2.5 \text { oz equiv. } \\
\text { per } 1,000 \text { kcals }\end{array}$ & $3.7(0-5)$ & $4.0(1-5)$ & $3.8(0-5)$ \\
\hline $\begin{array}{l}\text { Seafood and Plant } \\
\text { Proteins }\end{array}$ & 5 & $\begin{array}{l}\text { No Seafood or Plant } \\
\text { Proteins }\end{array}$ & $\begin{array}{l}\geq 0.8 \text { oz equiv. } \\
\text { per } 1,000 \text { kcals }\end{array}$ & $3.2(0-5)$ & $2.9(0-5)$ & $3.1(0-5)$ \\
\hline Fatty Acids $* *$ & 10 & $\begin{array}{l}\text { (PUFAs }+ \\
\text { MUFAs) / SFAs } \leq \\
1.2\end{array}$ & $\begin{array}{l}\text { (PUFAs + } \\
\text { MUFAs) / } \\
\text { SFAs } \geq 2.5\end{array}$ & $3.8(0-10)$ & $0.3(0-7)$ & $2.5(0-10)$ \\
\hline Refined Grains & 10 & $\begin{array}{l}\geq 4.3 \text { oz equiv. per } \\
1,000 \text { kcals }\end{array}$ & $\begin{array}{l}\leq 1.8 \text { oz equiv. } \\
\text { per } 1,000 \text { kcals }\end{array}$ & $6.0(0-10)$ & $5.5(0-10)$ & $5.8(0-10)$ \\
\hline Sodium & 10 & $\begin{array}{l}\geq 2.0 \text { grams per } \\
1,000 \text { kcals }\end{array}$ & $\begin{array}{l}\leq 1.1 \text { grams per } \\
1,000 \text { kcals }\end{array}$ & $9.0(0-10)$ & $9.0(5-10)$ & $9.0(0-10)$ \\
\hline Empty Calories & 20 & $\geq 50 \%$ of energy & $\begin{array}{l}\leq 19 \% \text { of } \\
\text { energy }\end{array}$ & $8.2(0-18)$ & $9.1(0-20)$ & $8.5(0-20)$ \\
\hline HEI-2010 Total score $* *$ & 100 & & & $55.8(33-77)$ & $50.3(31-73)$ & $53.7(31-77)$ \\
\hline
\end{tabular}

Abbreviations: HEI-2010, Healthy Eating Index, 2010; Equiv., equivalent; Kcals, kilocalories; PUFA, polyunsaturated fatty acid; MUFA, monounsaturated fatty acid; SFA, saturated fatty acid.

\footnotetext{
Independent T-test $p<0.03$

*** Independent T-test $p<0.0001$
}

Empty cell present because HEI-2010 score does not apply to these columns. 
Table 2.

Demographic and health characteristics of Challenge, Three Generation adolescents, and combined sample (N $=317$ )

\begin{tabular}{|c|c|c|c|}
\hline & Challenge $(n=196)$ & Three Generation $(n=121)$ & Combined sample $(\mathrm{N}=317)$ \\
\hline \multicolumn{4}{|l|}{ Sex } \\
\hline Males $(\%)$ & 49 & 0 & 31 \\
\hline Females $(\%)$ & 51 & 100 & 69 \\
\hline Age, mean (y) & 13.2 & 17.5 & 16.1 \\
\hline \multicolumn{4}{|l|}{ Weight status } \\
\hline BMI z-score ${ }^{a}$ & $0.7 \pm 1.15$ & $1.0 \pm 1.08 *$ & $0.83 \pm 1.13$ \\
\hline BMI $\% \geq 85^{\text {th }}$ percentile $(\%)$ & 12 & 22 & 27 \\
\hline BMI $\% \geq 95^{\text {th }}$ percentile (\%) & 27 & 35 & 16 \\
\hline \multicolumn{4}{|l|}{ Dietary Intake } \\
\hline Energy ${ }^{a}$ (kcals) & $2613 \pm 1136.3$ & $2394 \pm 1018.5$ & $2529 \pm 1096.4$ \\
\hline HEI total score & $62.8 \pm 11.75$ & $59.9 \pm 11.27 *$ & $61.7 \pm 11.64$ \\
\hline HEI-2010 total score & $55.8 \pm 8.23$ & $50.3 \pm 7.95 * *$ & $53.7 \pm 8.54$ \\
\hline $\operatorname{Iron}^{a}(\mathrm{mg} / \mathrm{d})$ & $16 \pm 7.9$ & $14 \pm 7.4 *$ & $16 \pm 7.8$ \\
\hline Fiber $^{a}(\mathrm{~g} / \mathrm{d})$ & $19 \pm 9.7$ & $17 \pm 9.0$ & $18 \pm 9.4$ \\
\hline Folate $^{a}(\mu \mathrm{g})$ & $446 \pm 217.2$ & $290 \pm 160.5^{* *}$ & $387 \pm 211.3$ \\
\hline Calcium $^{a}(\mathrm{mg} / \mathrm{d})$ & $1126 \pm 579.4$ & $907.5 \pm 500.7^{* *}$ & $1042 \pm 560.0$ \\
\hline Vitamin $A^{a}(\mathrm{IU})$ & $8253 \pm 5611.9$ & $7979 \pm 6330.1$ & $8148 \pm 5880.0$ \\
\hline Snack/desserts ${ }^{a}$ (servings/d) & $4.5 \pm 3.44$ & $3.4 \pm 2.34 * *$ & $4.1 \pm 3.11$ \\
\hline Sweetened beverages (soda) ${ }^{a}$ (servings/d) & $4.4 \pm 3.21$ & $4.9 \pm 2.96$ & $4.6 \pm 3.12$ \\
\hline
\end{tabular}

Abbreviations: y, years; BMI, body mass index; kcals, kilocalories; mg/d, milligrams per day; g/d, grams per day; $\mu$ g, micrograms; IU, international units; /d, per day.

\footnotetext{
${ }^{a}$ Numbers reported are means \pm standard deviation

Independent T-test $p<0.05$

**

Independent T-test $p<0.01$
} 
Table 3.

Correlations Between the HEI, HEI-2010, and Diet/Health Indicators

\begin{tabular}{|c|c|c|c|}
\hline \multirow[b]{3}{*}{ Iron $(\mathrm{mg} / \mathrm{d})$} & \multicolumn{3}{|c|}{$\underline{\text { Study Sample N }=317}$} \\
\hline & HEI & HEI-2010 & Fisher's R-Z $(\lambda)$ \\
\hline & $0.58^{* *}$ & $0.13^{*}$ & 6.66 \\
\hline Fiber $(\mathrm{g} / \mathrm{d})$ & $0.72^{* *}$ & $0.28^{* * *}$ & 7.77 \\
\hline Folate $(\mu \mathrm{g})$ & $0.65^{* *}$ & $0.27^{* *}$ & 6.25 \\
\hline Calcium, mg/d & $0.45^{* *}$ & -0.01 & 6.20 \\
\hline Vitamin A (IU) & $0.51^{* *}$ & $0.30^{* * *}$ & $3.17^{* * *}$ \\
\hline Snack/desserts (servings/d) & $0.40^{* *}$ & $-0.12^{*}$ & 6.82 \\
\hline Sweetened beverages (servings/d) & $0.18^{* *}$ & $-0.31^{* *}$ & 6.30 \\
\hline Energy (kcal) & $0.58^{* *}$ & -0.02 & 8.55 \\
\hline $\mathrm{BMI} \geq 85^{\text {th }}(\%)$ & -0.10 & -0.10 & 0 \\
\hline BMI $₫ 95^{\text {th }}(\%)$ & 0.02 & -0.03 & 0.63 \\
\hline Body fat ${ }^{1}(\%)$ & $-0.17^{*}$ & -0.02 & -1.31 \\
\hline Abdominal fat ${ }^{1}(\%)$ & $-0.19^{*}$ & -0.0004 & -1.66 \\
\hline Serum cholesterol $^{2}(\mathrm{mg} / \mathrm{dL})$ & $-0.28^{*}$ & -0.05 & -1.25 \\
\hline $\operatorname{HDL}_{\text {cholesterol }}^{3}(\mathrm{mg} / \mathrm{dL})$ & 0.03 & -0.03 & 0.31 \\
\hline $\mathrm{LDL}_{\text {cholesterol }}^{3}(\mathrm{mg} / \mathrm{dL})$ & $-0.27^{*}$ & -0.05 & -1.16 \\
\hline Impaired glucose tolerance ${ }^{4}(\mathrm{~T} 120$ glucose $>140 \mathrm{mg} / \mathrm{dL})$ & $-0.27^{*}$ & -0.15 & -0.65 \\
\hline
\end{tabular}

Abbreviations: HEI, Healthy Eating Index; HEI-2010, Healthy Eating Index-2010; Kcals, kilocalories; BMI, body mass index; HDL, high density lipoprotein; LDL, low density lipoprotein; mg/d, milligrams per day; g/d, grams per day; /d, per day; $\mu \mathrm{g}$, micrograms; IU, international units; $\mathrm{mg} / \mathrm{dL}$, milligrams per deciliter; T120, time at 120 minutes post glucose dose.

* $p<0.05$

** $p<0.006$

${ }^{1}$ Challenge subset only, $\mathrm{n}=153$

2 Challenge subset only, $\mathrm{n}=58$

${ }^{3}$ Challenge subset only, $\mathrm{n}=55$

${ }^{4}$ Challenge subset only, $\mathrm{n}=57$ 